\title{
Population Extinction and the Genetics of Adaptation
}

\author{
H. Allen Orr $^{\star}$ and Robert L. Unckless ${ }^{\dagger}$
}

Department of Biology, University of Rochester, Rochester, New York 14627

Submitted October 1, 2007; Accepted January 22, 2008;

Electronically published July 3, 2008

\begin{abstract}
AвSTRACT: Theories of adaptation typically ignore the effect of environmental change on population size. But some environmental challenges — challenges to which populations must adapt-may depress absolute fitness below 1, causing populations to decline. Under this scenario, adaptation is a race; beneficial alleles that adapt a population to the new environment must sweep to high frequency before the population becomes extinct. We derive simple, though approximate, solutions to the probability of successful adaptation (population survival) when adaptation involves new mutations, the standing genetic variation, or a mixture of the two. Our results show that adaptation to such environmental challenges can be difficult when relying on new mutations at one or a few loci, and populations will often decline to extinction.
\end{abstract}

Keywords: adaptation, beneficial mutation, extinction, probability of fixation.

Evolutionary theorists have turned their attention recently to the genetics of adaptation. These efforts have largely focused on identifying patterns that might characterize adaptation through DNA sequence space (reviewed in Orr 2005). This work has revealed several interesting patterns (Gillespie 1984; Orr 2002, 2005; Rozen et al. 2002) and has motivated a number of new experiments (Rozen et al. 2002; Sanjuan et al. 2004; Rokyta et al. 2005; Barrett et al. 2006; Kassen and Bataillon 2006). Nearly all work on the theory of adaptation, however, has been limited in at least one important way: it assumes that populations maintain a stable size following a sudden change in environment, a change to which the population must adapt.

In reality, however, some of the adaptations in which

* E-mail: aorr@mail.rochester.edu.

† E-mail: runckles@mail.rochester.edu.

Am. Nat. 2008. Vol. 172, pp. 160-169. (C) 2008 by The University of Chicago. $0003-0147 / 2008 / 17202-42890 \$ 15.00$. All rights reserved.

DOI: $10.1086 / 589460$ we are most interested-for example, antibiotic and insecticide resistance, adaptation to climate change, new diseases or parasites, and adaptation during colonization events-might involve changes in the physical or biological environment that are severe enough to cause a reduction in population size. If the population fails to adapt, it might well decline to extinction. This scenario, which obviously sits at the intersection of population genetics and ecology, differs fundamentally from that typically considered by population geneticists in that it is a race: adaptation must occur before the population becomes extinct (Maynard Smith 1989).

A number of previous studies have considered this problem. An especially large literature has analyzed adaptation of populations to an environment that changes gradually through time (see Lynch et al. 1991; Lynch and Lande 1993; Burger and Lynch 1995; Lynch 1996; Barton and Partridge 2000; Stockwell et al. 2003 and references therein). These models are typically quantitative genetic (polygenic) and consider a trait that is under stabilizing selection (intermediate trait values are optimal). However, as the environment changes through time and the optimal trait value shifts, natural selection drives phenotypic evolution. If this response to selection fails to keep up with the pace of environmental change, the population suffers an increasingly large lag load (Maynard Smith 1976) and may ultimately become extinct. (Small threatened populations may also be vulnerable to the accumulation of deleterious mutations; see Lynch [1996] for a review.)

A smaller body of work has considered the adaptation of a population to a sudden change in the environment, one that is sufficiently dramatic to decrease the absolute fitness of individuals to below 1 . Unable to replace itself, such a population will begin to decline geometrically (approximately exponentially) through time. The most important analyses of this problem are those of Gomulkiewicz and Holt (1995) and Holt and Gomulkiewicz (1997). In the first, Gomulkiewicz and Holt performed both population genetic and quantitative genetic analyses. Their approach was heuristic, and it contrasted the time required for a threatened population to reach a critical small density-a density at which extinction by demographic stochasticity is likely — with the time required for natural se- 
lection to adapt the population to the new environment. If the first time is less than the second, extinction is probable. In the second article, Holt and Gomulkiewicz (1997) expanded their analysis, using quantitative and population genetic (branching process) approaches to assess the effects of initial population size and initial frequency of favorable genetic variants on the probability of population extinction; they also considered the distribution of times to extinction.

Here we build on this previous work on adaptation to a sudden environmental change. Our approach differs from previous efforts in several ways. Most important, we consider the contributions to adaptation of new mutations as well as those from the standing genetic variation. Moreover, in the case of standing variation, we explicitly consider both previously deleterious and neutral variants. We restrict our attention to simple population genetic models as well as to a simple ecological model in which approximate analytic results are possible. In all cases, we calculate the probability that a population substitutes a beneficial allele of sufficiently large effect to escape extinction. Because we consider the fate of major mutations at one or several loci-not the cumulative effects of many genes that each have a modest effect-our results are most directly relevant to organisms such as microbes, in which a small number of genes can respond to an environmental challenge. Our results also bear on more complex organisms when only a small number of loci can respond biochemically to a particular environmental challenge. (For example, considerable evidence suggests that a small number of genes, perhaps only one, can confer resistance to the insecticide cyclodiene [ffrench-Constant et al. 2000].)

Our general conclusion is that adaptation to sudden environmental change is difficult, at least when using new mutations or previously deleterious alleles at one or a few loci. We discuss conditions under which this conclusion does not hold and the odds of successful adaptation improve.

\section{Model}

\section{Scenario and Analytic Approach}

It will help to begin by considering a deterministic version of our model. We consider a population that has discrete generations and no age structure. The population shows a simple pattern of regulation: population size $N$ increases or decreases geometrically unless the population is at carrying capacity $K$, in which case population size neither increases nor decreases. In general, population size at time $T=t$ is $N_{t}=N_{0} \bar{W}^{t}$, where $N_{0}$ is the initial population size and $\bar{W}$ is the mean absolute fitness of the population. This model—or, more exactly, its con- tinuous time analog — was introduced by MacArthur and Wilson (1967, chap. 4) and has been the subject of considerable work in the population ecology of extinction; for example, see Leigh (1981), Goodman (1987a, 1987b), and Lande (1993).

We consider a haploid population; later, we briefly consider diploids. We focus on a one-locus model with two alleles (or classes of physiologically equivalent alleles): the wild-type $A$ and the mutant $a$. We generally assume that the mutant type is initially either absent or rare. Before the change in environment, the population is able to replace itself and has large size $N_{0}$.

At time (generation) $T=0$, the environment suddenly changes, absolute fitness falls, and the population can no longer replace itself. In the new environment, the wildtype allele has absolute fitness $W_{A}=1-r$. (Note that because $r$ is positive, we break with convention and use $r$ to measure the rate of population decline; this unorthodox notation will simplify our presentation.) In the new environment, the mutant has absolute fitness $W_{a}=(1-$ $r)\left(1+s_{\mathrm{b}}\right) \approx 1+s_{\mathrm{b}}-r$, where $s_{\mathrm{b}}$ is a beneficial selection coefficient and the approximation assumes that $s_{\mathrm{b}}$ and $r$ are reasonably small. Because at time $T=0$ the population is composed entirely (or almost entirely) of wildtype individuals, $N$ on average decreases by a proportion $\sim r$ each generation. Unless the beneficial $a$ allele sweeps to high frequency reasonably quickly, the population will become extinct.

Real populations do not show deterministic growth. Instead, different individuals of the same genotype show chance differences in offspring number. This stochasticity affects, among other things, the probability that a beneficial mutation fixes in a population. Our approach, which takes this chance variation into account, is based on branching process theory. We use this theory to calculate the probability of fixation of a beneficial allele; this probability of fixation in turn lets us calculate the probability that a population escapes extinction by adapting. We focus only on beneficial mutations that show $s_{\mathrm{b}}-r>0$ because only such alleles increase absolute fitness above 1 (when taken singly) and so can save the population from extinction. As usual with branching process theory, the beneficial allele $a$ has two possible ultimate fates: it can become extinct, or it can increase in numbers without bounds. In the former case, the population will become extinct (as mean absolute fitness remains below 1); in the latter case, the population will survive (as the ultimate survival of an allele is equivalent to the ultimate survival of a population). Our assumption of unlimited growth of the $a$ lineage is obviously artificial. Real surviving lineages (and populations) must reach a finite equilibrium size $K$. This artificial aspect of our model—indeed, of any branching process model-is, however, not as serious a problem as 
it might seem. As has long been recognized (Harris 1963, pp. 10-11), we need assume only that once a lineage having $W_{a}>1$ reaches an appreciable size, it will almost certainly survive. The probability of reaching this critical size is, therefore, essentially equal to the probability of ultimate survival given by branching process theory. So long as this critical size is considerably smaller than $K$ (which is true with large $K$ ), branching process theory yields the correct probability of population survival.

In many of our analytic approximations, we assume that before the substitution of a beneficial mutation, population size declines deterministically through time, having size $N_{t}=N_{0}(1-r)^{t}$ at time $T=t$. Although this is obviously a simplification, it allows derivation of several useful, though approximate, results. We check all analytic results against exact computer simulations that account fully for variable offspring number and stochastic fluctuations in population size.

\section{Computer Simulations}

We performed forward computer simulations that were exact and that tracked a population that, at $T=0$, included $N_{0}=K-1$ individuals. Under usual branching process assumptions, individuals in the new environment reproduced independently, and mean offspring number per individual depended on genotype. In particular, in the new environment, wild-type individuals had a binomially (approximately Poisson) distributed number of surviving offspring with mean $W_{A}$, and mutant individuals had a binomially (approximately Poisson) distributed number of surviving offspring with mean $W_{a}$. Given enough time, population size will, as noted, either decline to 0 or grow without bound. In practice, a realization of our simulation ended in extinction if population size equaled 0 ; a realization ended in survival if the mutant allele $a$ was substituted and population size returned to its initial size of $N_{0}$. The proportion of realizations in which adaptation successfully occurred, preventing extinction, was recorded. Later, we consider alternative forms of population regulation; the relevant computer simulations are described there. All simulations were written in the language $R$ ( $R$ Development Core Team 2006).

\section{Results}

\section{New Mutations}

The probability that a new beneficial mutation that arises in generation $t$ goes to fixation and thereby prevents population extinction is 1 minus the probability that all such mutations are lost accidentally:

$$
P=1-(1-\Pi)^{N_{0} u(1-r)^{t}}
$$

where $\Pi$ is the probability of fixation of a new beneficial mutation and $N_{0} u(1-r)^{t}$ is the number of beneficial mutations that appear in generation $t$. We assume here that population size declines deterministically and that beneficial mutations are sufficiently rare to enjoy independent fates. From branching process theory, the probability that a new mutation fixes in a population that is declining exponentially is $\Pi \approx 2\left(s_{\mathrm{b}}-r\right)$. (See also Otto and Whitlock 1997.) Haldane's (1927) classic $\Pi \approx 2 s_{\mathrm{b}}$ approximation thus is reduced when a mutation arises in a population that is declining. With small to moderate probabilities of fixation, equation (1) becomes $P_{t} \approx 1-\exp \left[-2 N_{0} u\left(s_{\mathrm{b}}-\right.\right.$ $\left.r)(1-r)^{t}\right]$.

We can also calculate the cumulative probability that a mutation that appears by generation $t$ prevents extinction. Letting $A$ denote the cumulative number of mutations that appear by generation $t$ and noting that the number of mutations per generation falls as a geometric sequence, we have $A=N_{0} u \sum_{T=0}^{t}(1-r)^{T}=N_{0} u\left[1-(1-r)^{t+1}\right] / r$. To a good approximation, then, a mutation that appears by generation $t$ will save a population with probability

$$
\begin{aligned}
P_{T \leq t} & \approx 1-\left[1-2\left(s_{\mathrm{b}}-r\right)\right]^{A} \\
& \approx 1-\exp \left\{-\frac{2 N_{0} u\left(s_{\mathrm{b}}-r\right)\left[1-(1-r)^{t+1}\right]}{r}\right\} .
\end{aligned}
$$

Because most beneficial mutations arise in earlier rather than later generations, $P_{T \leq t}$ levels off with increasing $t$. Indeed, as $t$ becomes large $(t \rightarrow \infty)$ in equation (2), we can find the total probability that a new mutation will save the population. This probability is

$$
P_{\text {new }} \approx 1-\exp \left[-\frac{2 N_{0} u\left(s_{\mathrm{b}}-r\right)}{r}\right]
$$

When the term in brackets is small, this is, to a good approximation, $P_{\text {new }} \approx 2 N_{0} u\left(s_{\mathrm{b}}-r\right) / r$.

Equation (3) is our most important result. It shows that there is a ceiling on the chance that new mutations can save a declining population, and given reasonable parameter values, this ceiling is often low (see table 1). This reflects the fact that adaptation in a declining population is a race; adaptation must occur before a population becomes extinct. Worse, the challenge confronting the population pulls the rug out from under its rescue: the relevant environmental change causes the number of (potentially rescuing) beneficial mutations that appear each generation to systematically decrease. While our derivation of equation (3) rests on several approximations and simplifica- 
Table 1: Probability of population survival as predicted by analytic theory

\begin{tabular}{llllll}
\hline & $u=10^{-7}$ & $u=10^{-6}$ & $u=10^{-7}$ & $u=10^{-6}$ & $u=10^{-6}$ \\
& $s_{\mathrm{b}}=.03$ & $s_{\mathrm{b}}=.03$ & $s_{\mathrm{b}}=.03$ & $s_{\mathrm{b}}=.03$ & $s_{\mathrm{b}}=.04$ \\
& $r=.01$ & $r=.01$ & $r=.02$ & $r=.02$ & $r=.01$ \\
\hline$P_{\text {new }} .0040$ & .0392 & .0010 & .0100 & .0582 \\
$P_{\text {old (deleterious) }}$ a & .0022 & .0217 & .0014 & .0138 & .0273 \\
$P_{\text {old (neutral) }}{ }^{\mathrm{b}}$ & .0111 & .1052 & .0097 & .0929 & .1125 \\
$P_{\text {old (nearly neutral) }}$ & .0102 & .0977 & .0089 & .0852 & .1050 \\
\hline \multicolumn{2}{l}{ Note: $N_{0}=10,000 ; v=10^{-4}}$. \\
a $s_{\mathrm{d}}=0.01$. \\
${ }^{\mathrm{b}} N_{0} s_{\mathrm{d}}=0.5$.
\end{tabular}

tions, figure 1, which gives the results of exact computer simulations, shows that the derivation provides a good approximation to the probability of population survival, at least when $s_{\mathrm{b}}$ is of modest magnitude.

From equations (1) and (3), we can calculate each generation's contribution to the rescue of a population. Conditional on population survival, how often does a rescuing mutation arise in generation $T=0,1,2, \ldots$ ? Straightforward calculations show that

$$
P(T=t \mid \text { survival }) \approx r \exp (-r t) .
$$

This makes good sense; because the population declines approximately exponentially, the adaptive contribution of each generation must also decline approximately exponentially.

While we have, for simplicity, considered a one-locus model, it should be noted that the above results remain correct if $u$ is interpreted as the genomic rate of mutation to beneficial alleles of effect $s_{\mathrm{b}}$ (so long as $u$ remains small enough that mutations enjoy independent fates). Below, however, we return to our one-locus assumption.

\section{Previously Deleterious Alleles}

A population threatened by environmental change need not rely on new mutations to adapt. Instead, the population might respond by fixing alleles from the standing genetic variation. Here we consider one component of the standing variation: alleles that were previously definitely deleterious $\left(N_{0} s_{\mathrm{d}}>1\right)$ and that were maintained at mutation-selection balance.

Making the usual assumptions that the rate of mutation to the $a$ allele is $u$, that back mutation can be ignored, and that the $a$ allele suffers a deleterious effect $s_{\mathrm{d}}\left(s_{\mathrm{d}} \gg\right.$ $u), a$ segregates at an equilibrium frequency of $\hat{q} \approx u / s_{\mathrm{d}}$ in a haploid population. When the environment changes at time $T=0$, the $a$ allele becomes favorable and enjoys selective advantage $s_{\mathrm{b}}\left(s_{\mathrm{b}}\right.$ might be less than, greater than, or equal to $s_{\mathrm{d}}$ ). We temporarily ignore the contribution of new mutations and consider only the probability that these "old" alleles from mutation-selection balance save the population from extinction. Following our earlier logic, a population escapes extinction only if at least one of the $N_{0} \hat{q}$ copies of $a$ that segregate at time $T=0$ escapes accidental loss (Orr and Betancourt 2001). If these copies have independent fates, the population escapes extinction with probability

$$
\begin{aligned}
P_{\text {old }} & \approx 1-\exp \left[-2 N_{0} \hat{q}\left(s_{\mathrm{b}}-r\right)\right] \\
& \approx 1-\exp \left[\frac{-2 N_{0} u\left(s_{\mathrm{b}}-r\right)}{s_{\mathrm{d}}}\right] .
\end{aligned}
$$

Probability $P_{\text {old }}$ thus takes the same form as $P_{\text {new }}$, except that a term of $s_{\mathrm{d}}$ in the former replaces a term of $r$ in the latter. From $P_{\text {old }}$, it is easy to see that to ensure a $95 \%$

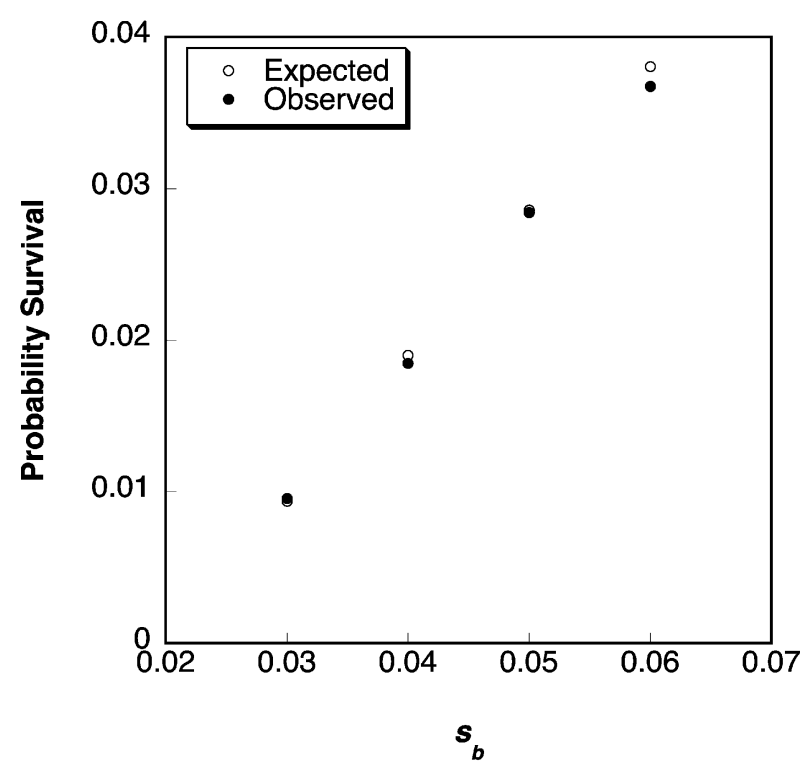

Figure 1: Probabilities of population survival from new mutations $\left(N_{0}=10^{4}, r=0.02, u=10^{-6}\right)$ over a range of $s_{\mathrm{b}}$, with no contribution from the standing genetic variation. Observed data based on 10,000 realizations of exact computer simulations. 


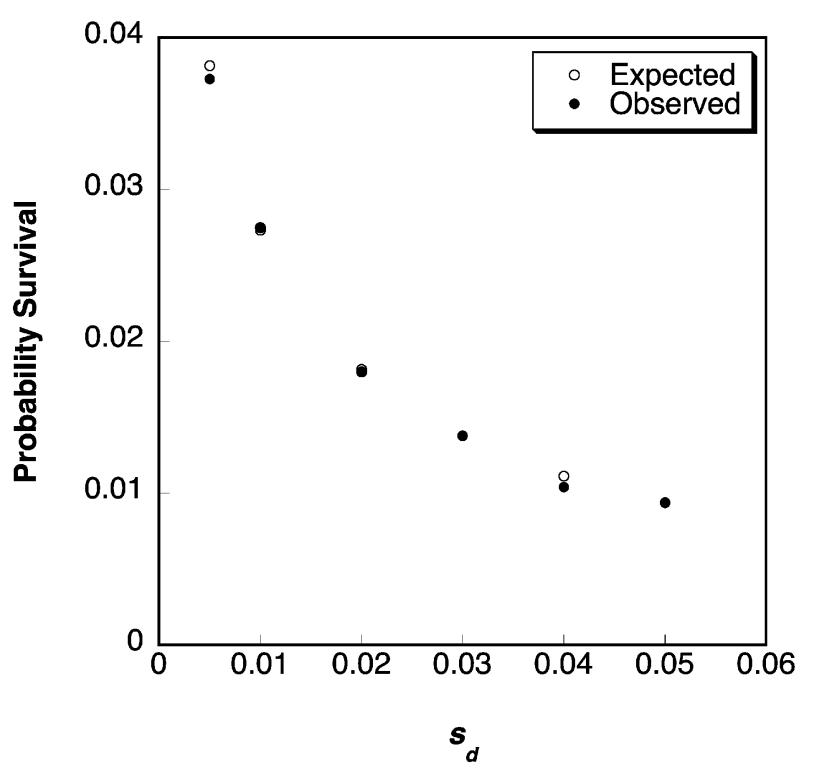

Figure 2: Probabilities of population survival from definitely deleterious $\left(N_{0} s_{\mathrm{d}}>1\right)$ standing genetic variation $\left(N_{0}=10^{4}, r=0.02, s_{\mathrm{b}}=0.05\right.$, $u=10^{-6}$ ) over a range of $s_{\mathrm{d}}$, with no contribution from new mutations. Observed data from 10,000 realizations of simulations.

probability of survival, a population must segregate for $N \hat{q} \approx 3 /\left[2\left(s_{\mathrm{b}}-r\right)\right]$ copies of the $a$ allele.

While simple, the above calculation's usefulness is limited by our assumption that the $a$ allele segregates at a deterministic frequency of $\hat{q} \approx u / s_{\mathrm{d}}$. In reality, $a$ will have a distribution of starting frequencies in finite populations. When back mutation can be ignored and selection against $a$ is reasonably strong, this stationary distribution at mutationselection-drift equilibrium is approximately gamma (Nei 1968):

$$
f(q)=\frac{\left(2 N_{0} s_{\mathrm{d}}\right) \exp \left(-2 N_{0} s_{\mathrm{d}} q\right)\left(2 N_{0} s_{\mathrm{d}} q\right)^{2 N_{0} u-1}}{\Gamma\left(2 N_{0} u\right)} .
$$

Integrating over this stationary distribution, the mean probability of population survival is

$$
\begin{aligned}
\bar{P}_{\text {old }} & =\int_{0}^{1} P_{\text {old }} f(q) d q \\
& =1-\left(\frac{s_{\mathrm{b}}-r+s_{\mathrm{d}}}{s_{\mathrm{d}}}\right)^{-2 N_{0} u} .
\end{aligned}
$$

This result is similar to one derived by Hermisson and Pennings (2005) for a diploid population (though they did not consider geometrically declining populations). Figure
2 shows that equation (5) provides an excellent approximation of the probability of population survival from the standing variation seen in computer simulations. Table 1 provides values of $\bar{P}_{\text {old }}$ from equation (5), given different combinations of parameter values.

We can also consider the case in which both "old" alleles from mutation-selection-drift balance and "new" alleles from mutations that arise after the environmental change can contribute to adaptation. In this case, a population might be saved by (i) alleles from the standing genetic variation, (ii) new mutations, or (iii) a mixture of the two. Following Hermisson and Pennings (2005), we simplify the analysis by defining rescue from the standing genetic variation if any of the haplotypes going to fixation segregated before the environmental change at $T=0$. Thus, the total probability of population survival is $P_{\text {total }}=$ $\bar{P}_{\text {old }}+\left(1-\bar{P}_{\text {old }}\right) P_{\text {new }}$, or

$$
P_{\text {total }} \approx 1-\left(\frac{s_{\mathrm{b}}-r+s_{\mathrm{d}}}{s_{\mathrm{d}}}\right)^{-2 N_{0} u} \exp \left(-2 N_{0} u \frac{s_{\mathrm{b}}-r}{r}\right)
$$

where we assume that the rate of mutation to the $a$ allele is the same before and after the environmental change. Figure 3 shows the probabilities of population survival from new and old mutations when both are allowed in computer simulations. Our analytic approximations again perform well.

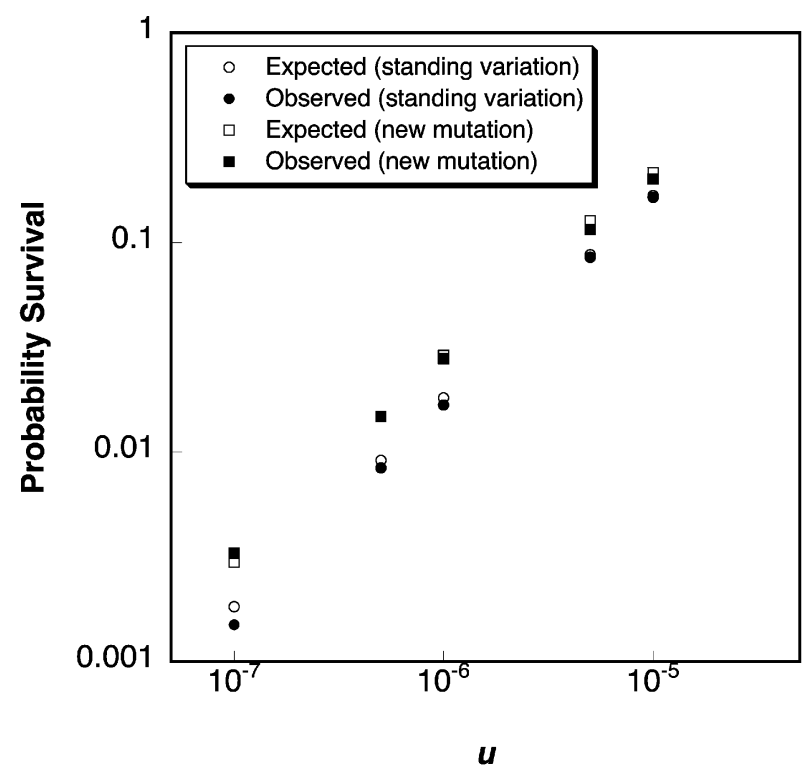

Figure 3: Probabilities of population survival from definitely deleterious standing genetic variation and new mutations $\left(N_{0}=10^{4}, r=0.02\right.$, $\left.s_{\mathrm{b}}=0.05, s_{\mathrm{d}}=0.02\right)$ over a range of $u(\log -\log$ plot). Observed data from 10,000 realizations of simulations. 
The fraction of cases in which the population escapes extinction by using the standing genetic variation (conditional on escaping extinction) is given by the ratio of equation (5) to equation (6). From this, it follows that previously deleterious alleles will rescue a population more often than will new mutations when the deleterious effects of $a$ were milder than

$$
\begin{aligned}
s_{\mathrm{d}}^{*} & <\frac{s_{\mathrm{b}}-r}{\left\{2-\exp \left[-2 N_{0} u\left(s_{\mathrm{b}}-r\right) / r\right]\right\}^{1 / 2 N_{0} u}-1} \\
& \approx \frac{s_{\mathrm{b}}-r}{\exp \left[\left(s_{\mathrm{b}}-r\right) / r\right]-1},
\end{aligned}
$$

where the approximation holds when $N_{0} u$ is small. Surprisingly, then, we can write down a simple solution to the conditions under which previously deleterious mutations are used more often than new mutations to save a declining population. Computer simulations confirm that the approximate form of equation (7) provides an excellent estimate of the observed threshold value of $s_{\mathrm{d}}^{*}$ (not shown).

\section{Previously Neutral Alleles}

A population might be rescued from extinction by substituting alleles that were previously neutral alleles and that became advantageous at time $T=0$. We first consider alleles that were strictly neutral and then turn to alleles that were nearly neutral.

Calculation of the probability that alleles from the strictly neutral standing variation rescue a population follows a logic similar to that with definitely deleterious alleles. The stationary distribution of $a$ now follows a beta distribution,

$$
f(q)=\frac{1}{B\left(2 N_{0} u, 2 N_{0} v\right) q^{2 N_{0} u-1}(1-q)^{2 N_{0} v-1}},
$$

where $B(x, y)=\Gamma(x) \Gamma(y) / \Gamma(x+y)$ and $v$ is the rate of back mutation to the $A$ allele (Crow and Kimura 1970). Integrating, we have

$$
\begin{gathered}
\bar{P}_{\text {old, neutral }}=\int_{0}^{1} P_{\text {old, neutral }} f(q) d q \\
\approx 1-M\left[2 N_{0} u, 2 N_{0}(u+v),-2 N_{0}\left(s_{\mathrm{b}}-r\right)\right],
\end{gathered}
$$

where $M[a, b, z]$ is the Kummer confluent hypergeometric function of the first kind (Abramowitz and Stegun 1970). Equation (8) is of biological interest only if $u \ll v$; on the other hand, if $u$ approaches $v$, the mean of the stationary distribution approaches $1 / 2$ and the population is inevi- tably (but trivially) rescued; the environmental change does not threaten the population because most individuals enjoy higher, not lower, fitness in the new environment. (More exactly, if $a$ begins at a high frequency, mean absolute fitness is $\bar{W}=1-r+q s_{\mathrm{b}}$ and the population need not decline at all.) Simulation data confirm that equation (8) is accurate over a range of what appear to be biologically plausible values of $u$ (fig. 4). Table 1 reports $\bar{P}_{\text {old, neutral }}$ from equation (8) given various combinations of parameter values.

If new mutations are allowed, the total probability that a population is rescued is given by equations ( 3 ) and (8), that is, by $\bar{P}_{\text {old, neutral }}+\left(1-\bar{P}_{\text {old, neutral }}\right) P_{\text {new }}$. As expected, a population is much more likely to be rescued if $a$ comes from the neutral standing variation than from either definitely deleterious standing variation or new mutations. The reason is that previously neutral alleles usually segregate at appreciable starting frequencies.

Turning to nearly neutral alleles, we must take into account both back mutation from $a$ to $A$ and the fitness effect of the slightly deleterious allele. For haploids, the stationary distribution of nearly neutral alleles is $f(q)=$ $c \times \exp \left[2 N_{0} s_{\mathrm{d}}(1-q)\right] \times q^{2 N_{0} u-1}(1-q)^{2 N_{0} v-1}$, where $c$ is a constant of integration that ensures that $f(q)$ integrates to 1 (Crow and Kimura 1970). Integrating $P_{\text {old }}$ over the stationary distribution of $q$, we find

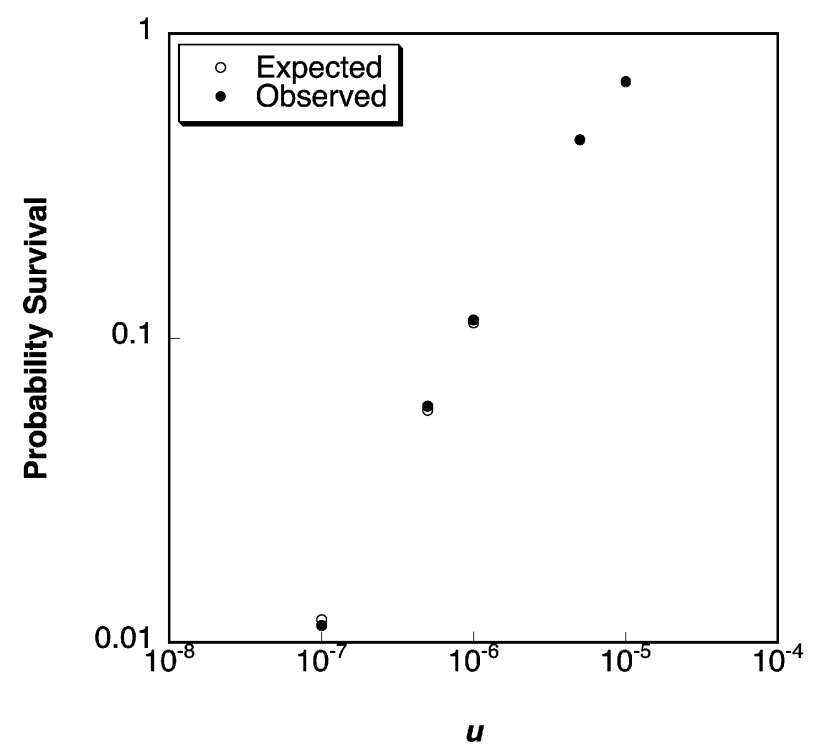

Figure 4: Probabilities of population survival from strictly neutral $\left(s_{\mathrm{d}}=0\right)$ standing genetic variation $\left(N_{0}=10^{4}, r=0.02, s_{\mathrm{b}}=0.05\right.$, $v=10^{-4}$ ) over a range of $u$, with no contribution from new mutations (log-log plot). Observed data from 10,000 realizations of simulations. 


$$
\begin{gathered}
\bar{P}_{\text {old, nearly neutral }} \approx \\
1-\frac{M\left[2 N_{0} u, 2 N_{0}(u+v),-2 N_{0}\left(s_{\mathrm{b}}-r+s_{\mathrm{d}}\right)\right]}{M\left[2 N_{0} u, 2 N_{0}(u+v),-2 N_{0} s_{\mathrm{d}}\right]} .
\end{gathered}
$$

Not surprisingly, this quantity falls between that found in the cases of strict neutrality and definitely deleterious alleles (see table 1). Simulations confirm that equation (9) is accurate over a wide range of parameter values (fig. 5).

\section{Diploidy and Multiple Loci}

In all the scenarios considered above-adaptation from new mutations or from previously deleterious or neutral alleles-our analysis can be extended trivially to diploids if we ignore the case of completely recessive alleles. If we define $s_{\mathrm{b}}$ and $s_{\mathrm{d}}$ as the heterozygous fitness effects of an allele-because heterozygous effects approximately determine both probabilities of fixation for beneficial alleles and mutation-selection balance frequencies for deleterious ones-the above equations remain correct if we replace $N_{0}$ with $2 N_{0}$ throughout. To take an example, the probability that a new mutation rescues a declining diploid population is

$$
P_{\text {new }} \approx 1-\exp \left[-\frac{4 N_{0} u\left(s_{\mathrm{b}}-r\right)}{r}\right] .
$$

This approach obviously assumes that an allele's beneficial heterozygous effect, $s_{b}$, is greater than $r$. Interestingly, this need not be the case. A population could be rescued (though with very low probability) even if an allele's heterozygous effect does not yield an absolute fitness above 1, so long as its homozygous effect does and the allele spreads to an appreciable enough frequency so that homozygotes are common. This is most likely when a beneficial allele arises from previously neutral or nearly neutral standing variation. In these cases, absolute copy number is relatively high.

Our analysis can also be extended trivially to the case of multiple loci. If there are $m$ loci-any one of which is capable of rescuing the population-the relevant probability of population survival, $P_{m}$, under any scenario (new mutations, previously deleterious alleles, etc.) is

$$
P_{m}=1-\prod_{i=1}^{m}\left(1-P_{i}\right)
$$

where $P_{i}$ is the probability of survival for the $i$ th locus under the relevant scenario. In the special case where the loci are all equivalent (exchangeable), equation (11) becomes $P_{m}=1-\left(1-P_{1}\right)^{m}$, where $P_{1}$ is the single-locus

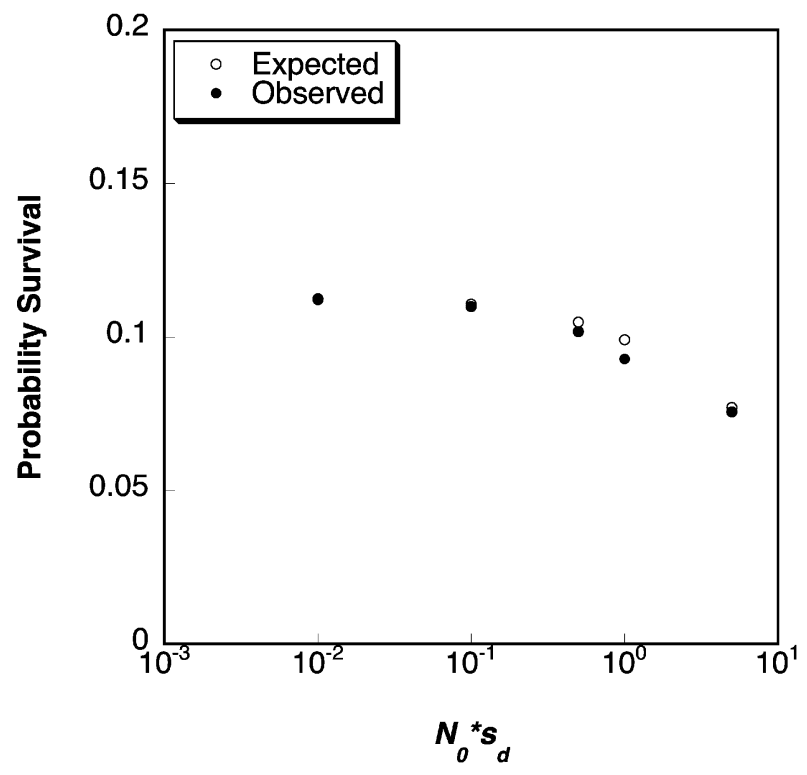

Figure 5: Probabilities of population survival from nearly neutral $(0<$ $\left.N_{0} s_{d}<1\right)$ standing genetic variation $\left(N_{0}=10^{4}, r=0.02, s_{\mathrm{b}}=0.05\right.$, $u=10^{-6}, v=10^{-4}$ ) over a range of $N_{0} s_{\mathrm{d}}$, with no contribution from new mutations (semilog plot). Observed data from 10,000 realizations of simulations.

probability of survival. Equation (11) obviously ignores epistatic interactions, which we do not pursue here. Equation (11) also ignores the (quantitative genetic) scenario in which no single locus can save the population but the cumulative effects of several to many might.

\section{Alternative Forms of Population Regulation}

The above theory assumes a particular model of population regulation: population size increases or decreases geometrically if $N<K$ and ceases to change if $N=K$. Although this simple model has been the subject of a number of theoretical studies of extinction, it is obviously somewhat artificial. We would like, therefore, to consider the effects of other, hopefully more realistic, models of population regulation on the genetics of adaptation in declining populations. To capture our biological problem, any such model must conform to two constraints: (1) wildtype absolute fitness must behave such that the population will become extinct deterministically unless a beneficial mutation sweeps to high frequency (thus $W_{A}<1$ at all $1 \leq N<K$ ), and (2) mutant absolute fitness must be $W_{a}=1$ when $N=K$, allowing any rescued population to reach an equilibrium size.

Although we were unable to make much analytic progress with such models, we studied several by computer simulation. We briefly describe two. In the first, the fit- 
nesses of both wild-type and mutant were logistic-like, such that absolute fitness gradually decreased as $N$ increased. Fitnesses were constructed, however, such that the wild-type and mutant alleles had a constant fitness difference. In particular, $W_{A}=1-r-\left(s_{\mathrm{b}}-r\right) N / K$ and $W_{a}=1+\left(s_{\mathrm{b}}-r\right)(1-N / K)$, so that $W_{a}-W_{A}=s_{\mathrm{b}}$ at all $N$, where $0 \leq N \leq K$. Mutant fitness here essentially follows the discrete logistic growth law considered by Roughgarden (1971) and Charlesworth (1971). In the second model, the fitness of the mutant again obeyed the discrete logistic growth law, whereas the wild-type was assumed to be unfit enough that it did not "feel" logistic regulation and had a constant fitness of $W_{A}=1-r$, with $0 \leq N \leq$ $K$. Note that at small $N$, both of these models collapse to our simple geometric one, as expected; small populations experience geometric growth. Note also that we consider mutations that affect only survival in the new environment, not the carrying capacity of the species; we assume that carrying capacity is set by factors other than those that the population is currently adapting to.

Forward computer simulations showed that under both models, probabilities of population survival were lower than those obtained under our earlier, geometric model (fig. 6). The reason is clear. With logistic-like regulation, absolute fitness decreases as $N$ nears $K$, lowering probabilities of mutant survival. But in a declining population, most beneficial mutations occur early on, when populations are large.

Though counterexamples might be possible, our exploration of alternative forms of population regulation (subject to our two constraints) suggests that our simple geometric model does not yield unusually dire results. Indeed, under some forms of regulation, population survival is less likely than indicated by our analytic findings.

\section{Conclusions}

Our analysis differs from previous ones in several ways. Most important, while a large literature considers adaptation in the face of continuous environmental change (e.g., Lynch et al. 1991; Lynch and Lande 1993; Burger and Lynch 1995), we consider a change that is abrupt. Our analysis also differs, however, from that of Gomulkiewicz and Holt (1995) and Holt and Gomulkiewicz (1997), who in prescient articles considered adaptation to a sudden and severe environmental change. There are several differences. First, as these authors emphasized, their analyses were mostly heuristic and considered the timescales on which demographic extinction versus response to selection occur. Second, their work focused on the time required for a threatened population to decline to a small critical density (say, 10-100 individuals); our approach features no such critical size. Third, Gomulkiewicz and Holt considered

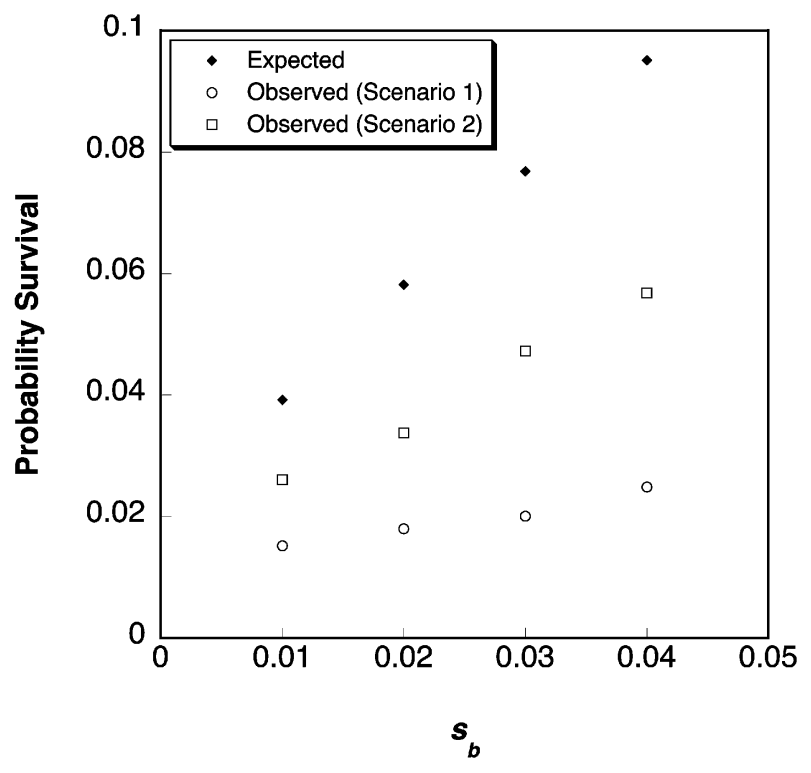

Figure 6: Probabilities of population survival from new mutations only $\left(N_{0}=10^{4}, r=0.01, u=10^{-6}\right)$ over a range of $s_{b}$, with density dependent fitness of both mutant and wild-type (scenario 1 ) or mutant only (scenario 2). Expected values are based on our standard (nondensity dependent) solution. Observed data from 10,000 realizations of simulations.

both polygenic and single-locus responses to selection, whereas we restrict our attention to single-locus models (or at least to those featuring a few major loci, any one of which might rescue a population). Last, Gomulkiewicz and Holt did not explicitly contrast the contributions of new versus previously deleterious versus neutral mutations to adaptation.

Despite these differences, our qualitative conclusion agrees with that of Gomulkiewicz and Holt; unless populations are very large or suffer only modest decreases in absolute fitness below 1, adaptation to a sudden environmental change can be difficult. This conclusion is especially clear when considering adaptation from new mutations or from alleles that were previously deleterious (see figs. 1, 2 ). We find that under these scenarios, the probability of population survival is often low, at least in single-locus models. The reason is clear. In both cases, the desired allele is present in low copy numbers in the critical early generations after the environmental change. Given the small probability that any particular copy of a beneficial allele will escape stochastic loss- $\Pi \approx 2\left(s_{\mathrm{b}}-r\right)$-population survival is difficult. Although this conclusion is perhaps unsurprising, it was not obvious that the probability of population survival would assume such simple analytic forms (see eqq. [3], [5]). (We further find that as $r$ becomes small, new mutations are more likely to rescue a population, because populations decline so slowly [eq. (7)].) In 
this case, previously deleterious alleles are unlikely to contribute to adaptation as often as new mutations unless $s_{\mathrm{d}}$ is so small as to approach nearly neutral values.) In any case, our results show that the scenarios typically favored by recent adaptation theorists-evolution from new mutations or from mutation-selection balance-may not involve rescue of a declining population unless $s_{\mathrm{b}}$ and/or $N_{0} u$ is large or $r$ and/or $s_{\mathrm{d}}$ is small. Again, we restrict attention here to cases where adaptation involves single mutations at one or a few loci.

Absolute copy number of an allele, not its frequency, determines the probability of population survival. Any factor that increases copy number will, therefore, increase the probability of survival. Our negative conclusion above will thus not hold under any scenario in which the initial copy number of a beneficial allele is increased. The most obvious such scenario involves alleles that were either strictly or nearly neutral before the environmental change and that became beneficial after. Table 1 confirms this intuition, showing that the probability of population survival is often high when considering previously neutral alleles. A key biological question is, therefore, how likely is it that an allele that had essentially no effect on fitness in the old environment will have a profound effect in the new environment, that is, will boost absolute fitness above 1 ?

Similarly, the probability of population survival increases nearly linearly with both population size and the number of (exchangeable) loci, even when adaptation uses new mutations or those that were previously deleterious. Both effects are intuitively obvious. Florida panthers are far less likely to survive a sudden environmental change than is a species of Drosophila (all else being equal) because panther populations are orders of magnitude smaller than Drosophila populations. Similarly, more loci (any one of which might save the population) provide more material for the genetic rescue of endangered populations and so help mitigate our negative, single-locus conclusions.

It is worth noting that our quantitative predictions are, in principle, testable. The most obvious tests would involve microbial experimental evolution work. Our findings for adaptation by new mutations (eq. [3]), for example, might be tested by placing a clone of microbes in a stressful environment (known $r$ ) and noting the proportion of replicates in which the population survives. Key parameters such as $N_{0}$ and $r$ could be manipulated experimentally, while parameters like $s_{\mathrm{b}}$ and $u$ could be measured in nonpermissive conditions (e.g., measuring the mutation rate to an antibiotic resistance allele). In organisms such as bacteriophage that have sufficiently small genomes, wholegenome sequencing could be used to confirm that adaptation occurred via single substitutions (e.g., Rokyta et al. 2005).

In closing, we stress that our analysis is limited in at least two ways. First, we have considered one biological scenario, that in which single mutations can increase absolute fitness above 1 and in which such mutations might then go to fixation in the threatened population. We have not considered the scenario in which populations survive because of modest allele frequency change at many genes, each having a small effect on fitness, and not because of substitution events at single loci. For this reason, as noted earlier, our results are perhaps most relevant to cases in which only one or a few genes can respond appropriately to an environmental challenge. Despite this caveat, Gomulkiewicz and Holt's (1995) results suggest that the probability of population survival can often remain low in quantitative genetic models, at least when population size is modest and environmental change abrupt. Second, our approach partly rests on branching process theory (though not entirely; see our discussion in "Alternative Forms of Population Regulation"), with its usual assumption of independent reproduction among individuals. While obviously an idealization, our approach at least yields an analytically tractable model, one that might serve as a baseline against which other, more complex models can be compared.

\section{Acknowledgments}

We thank J. Jaenike, L. Orr, D. Presgraves, and two anonymous reviewers for their comments on the manuscript. This work was supported by funds from the National Institutes of Health (GM51932) to H.A.O. and from the Robert and Mary Sproull Fellowship of the University of Rochester to R.L.U.

\section{Literature Cited}

Abramowitz, M., and I. A. Stegun. 1970. Handbook of mathematical functions. Dover, New York.

Barrett, R. D. H., R. C. MacLean, and G. Bell. 2006. Mutations of intermediate effect are responsible for adaptation in evolving Pseudomonas fluorescens populations. Biology Letters 2:236-238.

Barton, N., and L. Partridge. 2000. Limits to natural selection. BioEssays 22:1075-1084.

Burger, R., and M. Lynch. 1995. Evolution and extinction in a changing environment: a quantitative genetic analysis. Evolution 49:151163.

Charlesworth, B. 1971. Selection in density-regulated populations. Ecology 52:469-474.

Crow, J. F., and M. Kimura. 1970. An introduction to population genetics theory. Harper \& Row, New York.

ffrench-Constant, R. H., N. Anthony, K. Aronstein, T. Rocheleau, and G. Stilwell. 2000. Cyclodiene resistance: from molecular to population genetics. Annual Review of Entomology 48:449-466.

Gillespie, J. H. 1984. Molecular evolution over the mutational landscape. Evolution 38:1116-1129.

Gomulkiewicz, R., and R. D. Holt. 1995. When does evolution by natural selection prevent extinction? Evolution 49:201-207. 
Goodman, D. 1987a. Consideration of stochastic demography in the design and management of biological reserves. Natural Resource Modelling 1:205-234.

. 1987b. The demography of chance extinction. Pages 11-43 in M. E. Soulé, ed. Viable populations for conservation. Cambridge University Press, New York.

Haldane, J. B. S. 1927. A mathematical theory of natural and artificial selection. Pt. V. Selection and mutation. Proceedings of the Cambridge Philosophical Society 28:838-844.

Harris, T. E. 1963. The theory of branching processes. Prentice-Hall, Englewood Cliffs, NJ.

Hermisson, J., and P. S. Pennings. 2005. Soft sweeps: molecular population genetics of adaptation from standing genetic variation. Genetics 169:2335-2352.

Holt, R. D., and R. Gomulkiewicz. 1997. The evolution of species' niches: a population dynamic perspective. Pages $25-50$ in R. G. Othmer, F. R. Adler, M. A. Lewis, and J. C. Dallon, eds. Case studies in mathematical modelling: ecology, physiology, and cell biology. Prentice Hall, Englewood Cliffs, NJ.

Kassen, R., and T. Bataillon. 2006. The distribution of fitness effects among beneficial mutations prior to selection in experimental populations of bacteria. Nature Genetics 38:484-488.

Lande, R. 1993. Risk of population extinction from demographic and environmental stochasticity and random catastrophes. American Naturalist 142:911-927.

Leigh, E. G., Jr. 1981. The average lifetime of a population in a varying environment. Lournal of Theoretical Biology 90:213-239.

Lynch, M. 1996. A quantitative-genetic perspective on conversation issues. Pages 471-501 in J. C. Avise and J. L. Hamrick, eds. Conservation genetics: case studies from nature. Chapman \& Hall, London.

Lynch, M., and R. Lande. 1993. Evolution and extinction in response to environmental change. Pages 234-250 in P. M. Kareiva, J. G. Kingsolver, and R. B. Huey, eds. Biotic interactions and global change. Sinauer, Sunderland, MA.

Lynch, M., W. Gabriel, and A. M. Wood. 1991. Adaptive and demographic responses of plankton populations to environmental change. Limnology and Oceanography 36:1301-1312.
MacArthur, R. H., and E. O. Wilson. 1967. The theory of island biogeography. Princeton University Press, Princeton, NJ.

Maynard Smith, J. 1976. What determines the rate of evolution? American Naturalist 110:331-338.

. 1989. The causes of extinction. Philosophical Transactions of the Roval Society B: Biological Sciences 325:241-252.

Nei, M. 1968. The frequency distribution of lethal chromosomes in finite populations. Proceedings of the National Academv of Sciences of the USA 60:517-524.

Orr, H. A. 2002. The population genetics of adaptation: the adaptation of DNA sequences. Evolution 56:1317-1330.

2005. The genetic theory of adaptation: a brief history. $\mathrm{Na}-$ ture Reviews Genetics 6:119-127.

Orr, H. A., and A. J. Betancourt. 2001. Haldane's sieve and adaptation from standing genetic variation. Genetics 157:875-884.

Otto, S. P., and M. C. Whitlock. 1997. The probability of fixation in populations of changing size. Genetics 146:723-733.

R Development Core Team. 2006. R: a language and environment for statistical computing. R Development Core Team, Vienna.

Rokyta, D. R., P. Joyce, S. B. Caudle, and H. A. Wichman. 2005. An empirical test of the mutational landscape model of adaptation using a single-stranded DNA virus. Nature Genetics 37:441-444.

Roughgarden, J. 1971. Density-dependent natural selection. Ecology 52:453-468.

Rozen, D. E., J. A. G. M. de Visser, and P. J. Gerrish. 2002. Fitness effects of fixed beneficial mutations in microbial populations. Current Biology 12:1040-1045.

Sanjuan, R., A. Moya, and S. F. Elena. 2004. The distribution of fitness effects caused by single-nucleotide substitutions in an RNA virus. Proceedings of the National Academv of Sciences of the USA 101:8396-8401.

Stockwell, C. A., A. P. Hendry, and M. T. Kinnison. 2003. Contemporary evolution meets conservation biology. Trends in Ecology \& Evolution 18:94-101.

Associate Editor and Editor: Michael C. Whitlock 